OPEN ACCESS

Edited by: Riccardo Lencioni, University of Pisa, Italy

Reviewed by:

Francesco Tovoli,

University of Bologna, Italy

Dania Cioni,

University of Pisa, Italy

*Correspondence:

Tongsen Zheng

zhengtongsen@hrbmu.edu.cn Yang Zhou

zhouyang094@126.com

${ }^{+}$These authors have contributed equally to this work

Specialty section:

This article was submitted to Gastrointestinal Cancers: Hepato

Pancreatic Biliary Cancers,

a section of the journa

Frontiers in Oncology

Received: 16 September 2021 Accepted: 18 November 2021 Published: 09 December 2021

Citation:

Zhou M, Zhang C, Nie J, Sun $Y, X u Y$, Wu F, Huang $Y$, $L i$ S, Wang $Y$, Zhou $Y$ and Zheng $T$

(2021) Response Evaluation and Survival Prediction Following PD-1 Inhibitor in Patients With Advanced

Hepatocellular Carcinoma: Comparison of the RECIST 1.1, IRECIST, and mRECIST Criteria.

Front. Oncol. 11:764189. doi: 10.3389/fonc.2021.764189

\section{Response Evaluation and Survival Prediction Following PD-1 Inhibitor in Patients With Advanced Hepatocellular Carcinoma: Comparison of the RECIST 1.1, iRECIST, and MRECIST Criteria}

Meng Zhou ${ }^{1+}$, Chunhui Zhang ${ }^{1+}$, Jianhua $\mathrm{Nie}^{1}$, Yajuan Sun ${ }^{2}, \mathrm{Ye} \mathrm{Xu}^{2}$, Fangfang $\mathrm{Wu}^{1}$, Yuhong Huang ${ }^{1}$, Shun $L^{1}{ }^{1}$, Yuan Wang ${ }^{1}$, Yang Zhou ${ }^{2 *}$ and Tongsen Zheng ${ }^{1,3,4 *}$

\footnotetext{
${ }^{1}$ Department of Gastrointestinal Medical Oncology, Harbin Medical University Cancer Hospital, Harbin, China, ${ }^{2}$ Department of Radiology, Harbin Medical University Cancer Hospital, Harbin, China, ${ }^{3}$ Department of Phase 1 Trials Center, Harbin Medical University Cancer Hospital, Harbin, China, ${ }^{4}$ Key Laboratory of Molecular Oncology, Heilongjiang Cancer Institute, Harbin, China
}

Background: Precise evaluation of the efficacy of immunotherapy is critical in the effective management and treatment of advanced hepatocellular carcinoma (HCC). Therefore, the purpose of this study was to compare the response assessments achieved by different criteria and to evaluate the correlation between survival outcome and response assessment in HCC treated with programmed cell death protein 1 (PD-1) inhibitor.

Methods: Fifty patients with advanced HCC treated with first-line PD-1 inhibitor with baseline and follow-up CT images were analyzed. The patients were categorized into responders and nonresponders according to the criteria.

Results: When the response assessments between RECIST 1.1 and mRECIST were compared, no statistically significant differences were observed. Overall response rate was $16 \%$ by RECIST 1.1 and RECIST and was $24 \%$ by mRECIST. According to RECIST 1.1 and mRECIST, overall survival (OS) and progression-free survival (PFS) were not statistically different between the complete response (CR) and partial response (PR) groups and the stable disease (SD) and progressive disease (PD) groups. The OS and PFS were significantly different between responders and nonresponders according to mRECIST. The Cohen's Kappa for RECIST 1.1, iRECIST, and mRECIST was 0.534, 0.438 , and 0.363 , respectively.

Conclusion: The mRECIST criteria have a powerful ability to discriminate between responders and nonresponders and demonstrated significantly longer OS and PFS in responders than in nonresponders. However, mRECIST needs to be further improved in order for it to be widely used in the clinical evaluation of immunotherapy in HCC.

Keywords: immunotherapy, PD-1 inhibitor, advanced hepatocellular carcinoma, traditional evaluation criteria, immune-related criteria 


\section{INTRODUCTION}

Programmed cell death protein 1 (PD-1) expressed on $\mathrm{T}$ lymphocytes and activation of the receptors by their ligands (PDL1) prevents the reaction of $\mathrm{T}$ cells to tumor cells and thereby antitumor immunity (1-3). In recent years, additional antibodies targeting the $\mathrm{PD}-1 / \mathrm{PD}-\mathrm{L} 1$ have emerged as a promising treatment for inhibiting the progression, relapse, and metastasis of advanced hepatocellular carcinoma (HCC) (4). The initial effect of an PD-1/ PD-L1 inhibitor can result in an increase in tumor diameter caused by immune cell infiltration (5). The coexistence of immune cell infiltration and chronic inflammation in HCC might develop an atypical response pattern and patients may initially meet the criteria for progressive disease (PD) but later show stable or reduced tumor burden (6). This can result in failure of the conventional criteria, named Response Evaluation Criteria in Solid Tumors 1.1 (RECIST 1.1) (7), to capture the atypical patterns of tumor response. To address this issue, modified RECIST (mRECIST) $(8,9)$ and immunotherapy modified Response Evaluation Criteria in Solid Tumors (iRECIST) (10) were introduced, aiming to assist with discrimination between $\mathrm{PD}$ and pseudoprogression (PSPD). mRECIST only measures the arterially enhanced parts of the HCC target lesions. iRECIST is based on RECIST 1.1. The major difference between RECIST 1.1 and iRECIST is that the lesion categorized as PD in RECIST 1.1 requires verification by subsequent examinations to distinguish PD from PSPD. A study assessed the efficacy of nivolumab in patients with advanced HCC who achieved an overall response rate (ORR) of $18 \%$ by mRECIST and $14 \%$ by RECIST 1.1 following second-line treatment (11). Simultaneously, many studies have shown that the conventional criteria (RECIST 1.1) underestimated the benefits of immunotherapy in solid tumors, which may cause an early discontinuation of immune treatment (12-14). In addition, the ORR by mRECIST was regarded as a dependent predictor of overall survival (OS) in HCC treated with targeted therapy, meaning that responders survive significantly longer than nonresponders (15-19). However, there was no consensus on which response evaluation criteria were superior in immunotherapy of HCC, which led to complexities in patient management in different clinical trials. Additionally, the comparisons between the current standard (RECIST 1.1, iRECIST, and mRECIST) assessments are inadequate in advanced HCC. The evidence regarding the association of OS and response assessment by RECIST 1.1, iRECIST, and mRECIST in HCC treated with PD-1 inhibitor is scarce, especially in real-world settings.

The purpose of this study was to compare the response assessments using RECIST 1.1, mRECIST, and iRECIST and to evaluate the correlation between survival outcome and response assessment achieved by these criteria in HCC treated with PD1 inhibitor.

\section{MATERIALS AND METHODS}

\section{Patients}

Patients with advanced HCC receiving PD-1 inhibitor were retrospectively enrolled at the Harbin Medical University Cancer Hospital, China, from March 2017 to September 2020. Eligibility for this research included a diagnosis of HCC and the dosing and treatment duration of PD-1 inhibitor decided according to standard guidelines and treating physicians' judgment. Patients should also have undergone baseline computed tomography (CT) scans within 1 month before immune treatment and a follow-up CT scan within 3 months of the last dose of immunotherapy. The main exclusion criteria included the following: patients previously treated with an anti-PD-1, anti-PD-L1, anti-CTLA-4, or other agents targeting Tcell costimulation or immune checkpoint pathways; patients treated with other immunosuppressive medication within 2 weeks before starting PD-1 inhibitor treatment; patients with inadequate radiological evaluation (without an initial CT scan before/after immunotherapy or poor image quality); patients with no target lesions for analysis; and incomplete patient data caused by early death or lost to follow-up (Figure 1).

Baseline data at starting PD-1 inhibitor treatment included age, gender, hepatitis B virus (HBV), tumor markers [alpha fetoprotein (AFP)], and types of PD-1 (Table 1). This study was approved by the Ethics Review Board of Harbin Medical University. The requirement for informed consent from the patients was waived due to the retrospective design of this study, and patient information was protected.

\section{Image Analysis}

All contrast-enhanced dual-phase CT examinations of the chest, abdomen, and pelvis were performed on a 64-section multidetector CT scanner (128-slice, Siemens Medical System, Erlangen, Germany). The scanning parameters were set as follows: slice thickness and reconstruction interval, $1.25 \mathrm{~mm} ; 120 \mathrm{kV}, 250-300$ $\mathrm{mAs}$; a matrix of $512 \times 512$; image reconstruction, $1 \mathrm{~mm}$. To obtain contrast-enhanced image data, a nonionic iodinated contrast agent (iodine concentration: $350 \mathrm{mg} / \mathrm{ml}$ ) was administered intravenously by a contrast injector at a speed of $4 \mathrm{ml} / \mathrm{s}$. The dynamic contrastenhanced imaging data were obtained after unenhanced abdominal

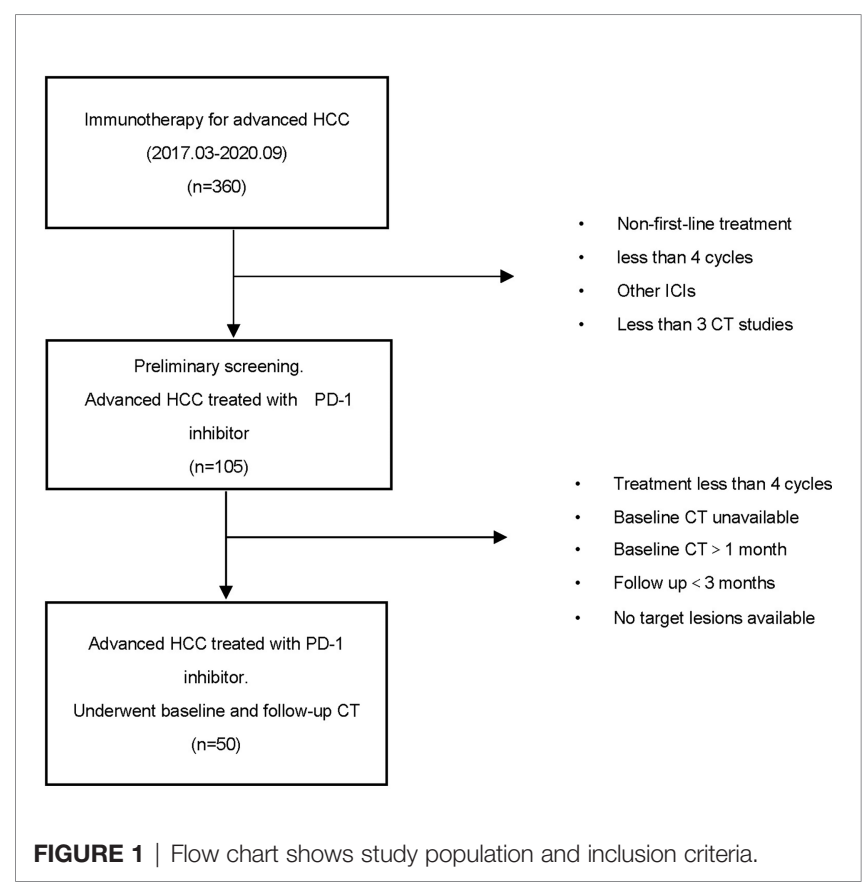


TABLE 1 | General characteristics of the patients, immunotherapy, and CT studies.

\begin{tabular}{|c|c|c|}
\hline Characteristic & Level & Value \\
\hline \multirow{2}{*}{$\begin{array}{l}\text { Age (years, median (IQR)) } \\
\text { Gender }(n(\%))\end{array}$} & & $54(40-70)$ \\
\hline & Male & $3(6)$ \\
\hline \multirow[t]{2}{*}{ ECOG $(n(\%))$} & 0 & $47(94)$ \\
\hline & 1 & $3(6)$ \\
\hline BCLC stage $(n(\%))$ & $\mathrm{B}$ & $16(32)$ \\
\hline $\operatorname{AFP}(n(\%))$ & $\leq 400 \mathrm{ng}$ & $29(58)$ \\
\hline \multirow[t]{2}{*}{ Drinking history (n (\%)) } & Yes & $12(24)$ \\
\hline & No & $38(76)$ \\
\hline \multirow[t]{2}{*}{ Smoking history (n (\%)) } & Yes & $17(34)$ \\
\hline & No & $33(66)$ \\
\hline HBV history ( $(\%))$ & Yes & $33(66)$ \\
\hline Extrahepatic spread status ( $(\%))$ & No & $18(36)$ \\
\hline \multirow[t]{5}{*}{ PD-1 inhibitor (n (\%)) } & Nivolumab & $8(16)$ \\
\hline & Pembrolizumab & $12(24)$ \\
\hline & Camrelizumab & $10(20)$ \\
\hline & Tislelizumab & $18(36)$ \\
\hline & Sintilimab & $2(4)$ \\
\hline & & 2-8 (median: 3) \\
\hline \multirow[t]{2}{*}{ Further systemic treatments $(n(\%))$} & Yes & $37(74)$ \\
\hline & No & $13(26)$ \\
\hline
\end{tabular}

HBV, hepatitis B virus; ICl, immune checkpoint inhibitor.

scanning. We obtained the arterial phase, the portal vein phase, and the equilibrium phase at approximately $30-33,67-70$, and $177-180$ s, respectively.

A central radiological review of imaging was evaluated by two radiologists (with 10 and 5 years of experience in CT reading, respectively) independently. If the opinions were not uniform, a third radiologist with 15 years of experience blinded to the results assessed by the others, re-evaluated the images, and disagreements were resolved by discussion. All of the radiologists blinded to the outcome of the patients.

\section{Response Assessment}

As per RECIST 1.1, iRECIST, and mRECIST, the radiologists reviewed the imaging to determine target tumor lesions, measure sum of diameters, assess nontarget lesions, and identify/measure new lesions. All of the radiologists blinded to the outcome of the patients, and then, they analyzed the image information according to RECIST 1.1, iRECIST, and mRECIST finally. Comparing the baseline and follow-up CT images, response to immunotherapy was classified into four categories according to RECIST 1.1 and mRECIST: complete response (CR), partial response (PR), stable disease (SD), and progressive disease (PD). Immunotherapy assessed by iRECIST was classified into immune CR (iCR), immune PR (iPR), immune SD (iSD), immune-unconfirmed PD (IUPD), and immuneconfirmed PD (ICPD). A first PD was assessed by RECIST 1.1, followed by any non-PD response (IUPD) by iRECIST. An ICPD was evaluated on follow-up imaging 4-8 weeks after IUPD. The patients were categorized into responders (CR or PR) and nonresponders (SD or PD) according to RECIST 1.1 and mRECIST. The ORR was defined as CR plus PR rates.

The association between the clinical outcome measure (OS, PFS) and response evaluation were analyzed by an experienced gastrointestinal oncology clinician (with 5 years of experience) who specialized in the diagnosis and treatment of HCC. In addition, the clinician blinded to the outcome of the patients. The OS was defined from the baseline date to the date for all-cause mortality. Progression-free survival (PFS) was defined as the time from the start of PD-1 to the progression of tumors (in any aspect) or death (for any reason). The best overall response (BOR) per RECIST and immune BOR were also assessed. They were obtained with all tumor assessments after initiation of PD-1 inhibitor until documented PD according to the respective criteria.

\section{Statistical Analysis}

Normally distributed continuous variables were expressed as mean \pm standard deviation (SD). Independent $t$-tests were performed for continuous independent variables. Cohen's Kappa test was used to assess the intra- and interobservational reliability between the two radiologists. The coefficients between 0.00 and 0.20 , indicated slight agreement; 0.21 and 0.40 , fair agreement; 0.41 and 0.60 , moderate agreement; 0.61 and 0.80 , substantial agreement; and 0.81 and 1.00 , almost perfect agreement. Survival analysis curves were drawn using the Kaplan-Meier method, and the Gehan-Breslow-Wilcoxon test was used for comparisons. All statistical analyses were performed using SPSS software (version 22.0, IBM). $p<0.05$ was considered statistically significant in all comparisons. 


\section{RESULTS}

\section{Response Assessment by RECIST 1.1 and iRECIST}

The response assessment of CR/iCR $(n=1), \mathrm{PR} / \mathrm{iPR}(n=7), \mathrm{SD} /$ iSD $(n=7)$, and ORR $(16 \%)$ by RECIST 1.1 were in common with iRECIST. Discordance between iRECIST and RECIST 1.1 was observed in the assessment of PD. Thirty-five patients (70\%) were considered to have PD as per RECIST 1.1 while the disease was considered ICPD $(25,50 \%)$ and IUPD (10, 20\%) as per iRECIST (Table 2). Ten patients were categorized as having IUPD as subsequent scans were unable to be performed during the follow-up period. Each pair was grouped together, leading to two categories: responders (CR and PR) and nonresponders (SD and PD). The response assessment of the two categories was the same between RECIST 1.1 and iRECIST (Table 3; Figure 2).

The ORR (16\%) by RECIST 1.1 was the same as that by iRECIST (Table 2). The BOR by RECIST 1.1 and iRECIST was reported in Table 4. The differences in BOR between RECIST 1.1 and iRECIST evaluation were also found in the assessment of SD ( $n=16,32 \%$ vs. $n=15,30 \%)$ and $\mathrm{PD}(n=21,42 \%$ vs. $n=15$, $30 \%)$. The distribution of response assessment and BOR assessment by RECIST 1.1 and iRECIST is shown in Figure 3.

\section{Response Assessment by RECIST 1.1 and MRECIST}

Fifteen patients were considered to have an immune response (1 additional CR, 7 additional PR, and 7 additional SD) as per RECIST 1.1 (Table 2). Compared with RECIST 1.1, 21 (42\%) patients who achieved CR $(n=6,12 \%), \mathrm{PR}(n=6,12 \%)$, and SD $(n=9,18 \%)$ were assessed by mRECIST. However, when

TABLE 2 | Treatment outcomes per RECIST 1.1, iRECIST, and mRECIST.

\begin{tabular}{lccc}
\hline $\mathbf{N}=\mathbf{5 0}$ & RECIST 1.1 & iRECIST & mRECIST \\
\hline Response No. (\%) & & & \\
CR/iCR & $1(2)$ & $1(2)$ & $6(12)$ \\
PR/iPR & $7(14)$ & $7(14)$ & $6(12)$ \\
SD/iSD & $7(14)$ & $7(14)$ & $9(18)$ \\
PD/ICPD & $35(70)$ & $25(50)$ & $29(58)$ \\
IUPD & & $10(20)$ & $12(24)$ \\
ORR & $8(16)$ & $8(16)$ &
\end{tabular}

CR, complete response; iCR, immune-complete response; $P R$, partial response; $i P R$, immune-partial response; $S D$, stable disease; iSD, immune-stable disease; $P D$, progressive disease; iPD, immune-progressive disease; ICPD, immune-confirmed $P D$; IUPD, immune-unconfirmed PD; ORR, overall response rate. we compared the response assessment between RECIST 1.1 and mRECIST, no statistically significant differences were found $(p>0.05)$ (Table 3 ).

The ORR was $24 \%$ by mRECIST for anti-PD- 1 antibodytreated patients, with a slight difference $(8 \%)$ between RECIST $1.1(16 \%)$ and mRECIST. The BOR by RECIST 1.1 and mRECIST is reported in Table 4. The discordance between RECIST 1.1 and mRECIST evaluation was most common for the BOR assessment of CR ( $n=0,0 \%$ vs. $n=8,16 \%), \mathrm{PR}(n=13$, $26 \%$ vs. $n=9,18 \%)$ and $\mathrm{PD}(n=21,42 \%$ vs. $n=18,36 \%)$. The distribution of response assessment and BOR assessment by RECIST 1.1 and mRECIST is shown in Figure 3.

\section{Correlation of Response Categories With OS}

The mean OS of the study population was $20.9 \pm 12.5$ months (range: 2 to 45 months) based on follow-up data. The 6-, 12-, 18-, and 24 -month OS rates were $88 \%, 74 \%, 50 \%$, and $40 \%$, respectively. Figure 4 demonstrates the OS rates in the response and nonresponse groups according to RECIST 1.1 and mRECIST.

The OS of anti-PD-1 antibody-treated patients defined by RECIST 1.1 and iRECIST was the same. According to RECIST 1.1 and mRECIST, the OS was not statistically different between the CR and PR groups $(p>0.05)$, and between the SD and PD groups $(p>0.05)$ (Table 5). According to RECIST 1.1, the mean OS was $25.5 \pm 11.1$ months among responders and $20.7 \pm 13.0$ months among nonresponders ( $p=0.458)$ (Table 5). According to mRECIST, the mean OS was $28.8 \pm 10.2$ months among responders and $19.0 \pm 12.5$ months among nonresponders $(p=$ 0.018) (Table 5). Consequently, the OS was significantly different between responders and nonresponders according to mRECIST $(p<0.05)$. However, there were no significant differences when we compared OS between responders and nonresponders grouped by RECIST 1.1. The differences in OS between responders and nonresponders according to RECIST 1.1 and mRECIST are illustrated in Figure 5.

\section{Correlation of Response Categories With PFS}

The mean PFS of the study population was $11.5 \pm 10.2$ months based on follow-up data. The PFS of HCC patients defined by RECIST 1.1 was the same with iRECIST. The PFS were not statistically different between the CR and PR groups $(p>0.05)$ based on RECIST 1.1 and mRECIST, as well as between the SD and PD groups $(p>0.05)$ (Table 5). According to RECIST 1.1, the mean PFS was $19.8 \pm 12.7$ months among responders and

TABLE 3 | The results of univariable analysis comparing RECIST 1.1, iRECIST, and mRECIST criteria.

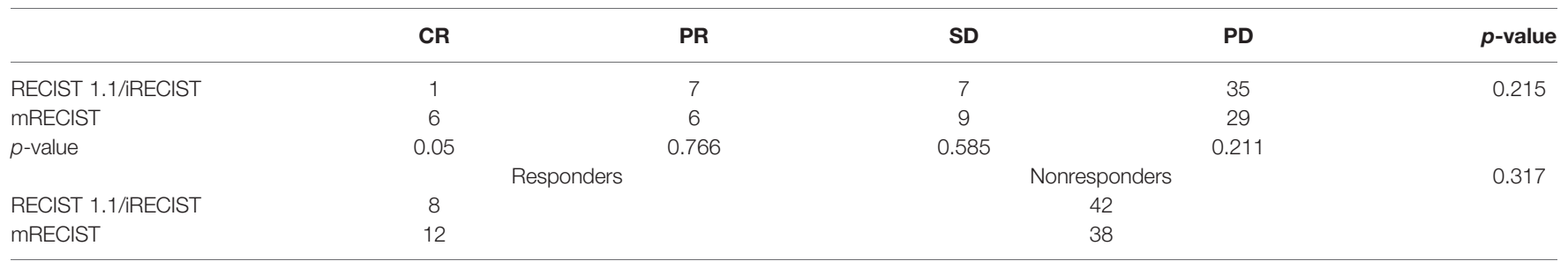

$C R$, complete response; $P R$, partial response; $S D$, stable disease; $P D$, progressive disease. 

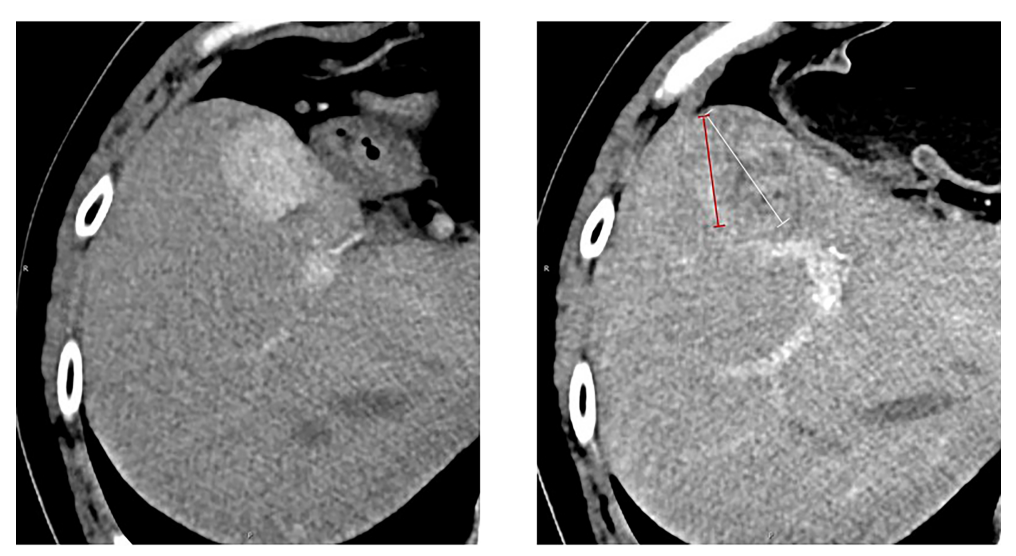

FIGURE 2 | Measurement of the longest tumor diameter in a target hepatic lesion: mRECIST vs. RECIST 1.1. Arterial-phase CT scan obtained after immunotherapy. According to RECIST 1.1, the overall longest diameter of the tumor is captured (white arrow), regardless of the presence of a large area of intratumoral treatmentinduced necrosis. In contrast, mRECIST measurement (red arrow) only includes the longest diameter of the viable portion of the tumor, as recognized by contrast enhancement. A 54-year-old man who had an arterial-phase CT examination before and after 27 cycles of immunotherapy. The baseline CT size of the target hepatic lesion was $30 \mathrm{~mm}$ (left). After immunotherapy, follow-up CT (right) showed treatment response of previous lesions. The response was assessed as PD according to RECIST 1.1 (47 mm) and SD based on mRECIST (34 mm).

$9.9 \pm 9.1$ months among nonresponders $(p=0.011)$ (Table 6). The mean PFS was $18.7 \pm 10.8$ months among responders and $9.2 \pm 9.1$ months among nonresponders $(p=0.004)$ (Table 6) assessed by mRECIST. Accordingly, the PFS were significantly different between responders and nonresponders according to mRECIST $(p<0.05)$. In addition, the PFS was also statistically different between these two groups according to RECIST $1.1(p<$ $0.05)$. The specific differences in PFS between responders and nonresponders according to RECIST 1.1 and mRECIST are illustrated in Figure 5.

\section{Comparison of the Response Assessment of the Two Radiologists}

Intrareader variability for all criteria is summarized in Table 7. Cohen's Kappa for RECIST 1.1, iRECIST, and mRECIST was 0.534 (95\% CI: 0.305-0.763), 0.438 (95\% CI: 0.426-0.450), and 0.363 (95\% CI: $0.351-0.375)$, respectively. Overall, there was moderate agreement for RECIST 1.1 and iRECIST and fair agreement for mRECIST.

TABLE 4 | Best overall response according to RECIST 1.1, iRECIST, and mRECIST criteria.

\begin{tabular}{lccc}
\hline $\mathbf{N}=\mathbf{5 0}$ & RECIST 1.1 & iRECIST & mRECIST \\
\hline Best response No. (\%) & & & \\
CR/iCR & $0(0)$ & $0(0)$ & $8(16)$ \\
PR/iPD & $13(26)$ & $13(26)$ & $9(18)$ \\
SD/iSD & $16(32)$ & $15(30)$ & $15(30)$ \\
PD/ICPD & $21(42)$ & $15(30)$ & $18(36)$ \\
IUPD & & $7(14)$ & $17(34)$ \\
CR or PR & $13(26)$ & $13(26)$ &
\end{tabular}

(i)CR, (immune-)complete response; $P R$, (immune-)partial response; $S D$, (immune-)stable disease; $P D$, (immune-)progressive disease; ICPD, immune-confirmed PD; IUPD, immune-unconfirmed $P D$.

\section{DISCUSSION}

Recent immune therapies, including immune checkpoint inhibitors, have shown encouraging clinical results in patients with advanced HCC $(20,21)$. Programmed death receptor-1 (PD-1)/PD-1 ligand (PD-L1) inhibitors, such as nivolumab and pembrolizumab, have been conditionally approved by the US Food and Drug Administration for second-line treatment of advanced HCC $(4,22)$. In addition, a number of criteria, including traditional
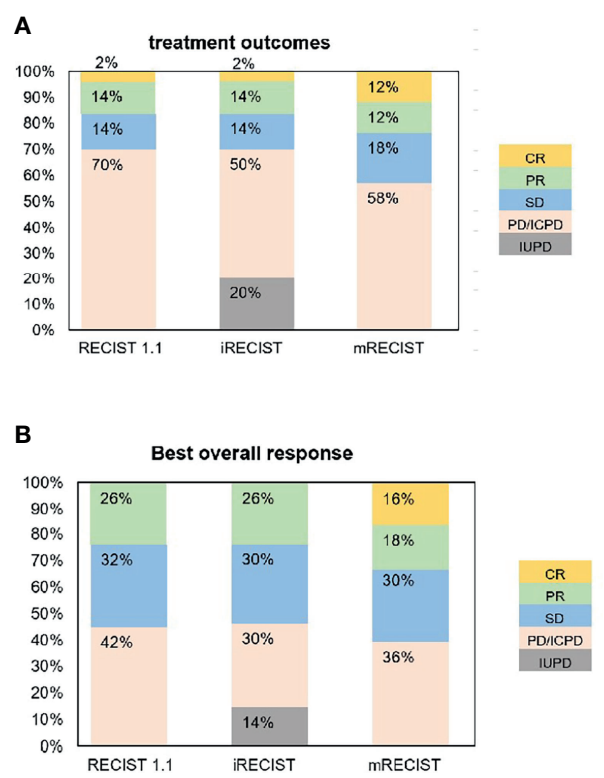

FIGURE 3 | Treatment outcomes (A) and best tumor response (B) per RECIST 1.1, iRECIST, and mRECIST. 

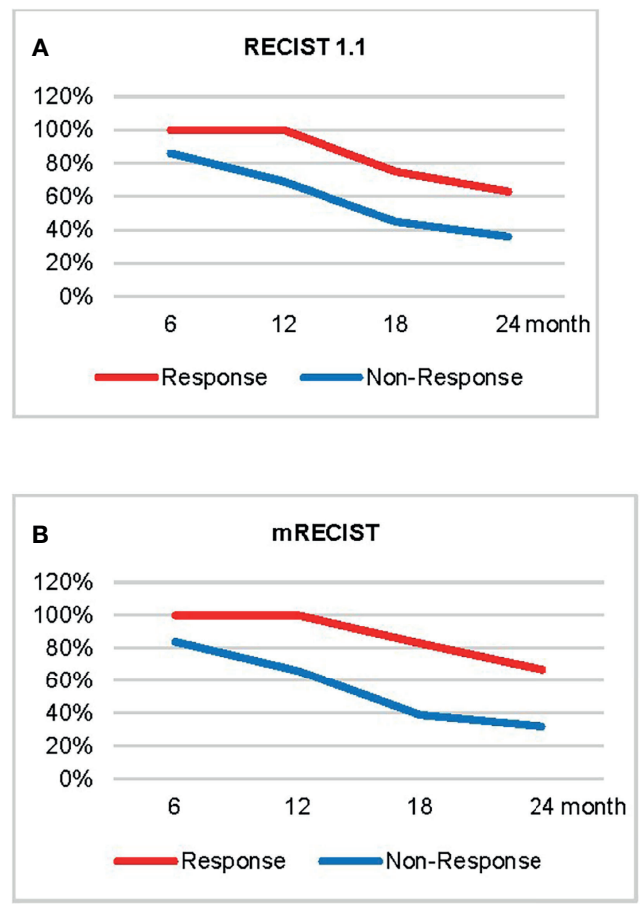

FIGURE 4 | The overall survival rate in different response groups according to RECIST 1.1 (A) and mRECIST (B).

evaluation criteria (RECIST 1.1) and immune-related criteria (iRECIST and mRECIST), have been reported to assess the efficacy of immunotherapy. However, it is unclear which criteria are best for evaluating immune-related response. In addition, the correlations between survival outcome and response assessment achieved by conventional and immunotherapy-modified methods in patients with HCC are also unknown.

This study explored a longer ORR and a greater number of BOR by mRECIST compared with the other criteria. A significant difference between responders and nonresponders assessed by mRECIST $(p<0.05)$ was observed. Our study showed a significantly longer OS and PFS in responders than nonresponders assessed by mRECIST than traditional evaluation criteria $(p<0.05)$.

The tumor assessment by RECIST1.1 is more rigorous and accurate than mRECIST. Patients who have achieved complete tumor remission by mRECIST were responding to immunotherapy actually. Thus, immunotherapy for those patients will be expected to extend the patient's OS. This means that the mRECIST standard actually prolongs the survival period of some patients who "cannot benefit from immunotherapy". Furthermore, the intrareader variability of mRECIST was lower than that of the other criteria evaluated in this study and may explain why mRECIST is not widely used in clinical applications and indicates that mRECIST needs to be improved.

Previous studies have shown that conventional criteria underestimated the ORR for immunotherapy by up to 15\% (2326). Simultaneously, it achieved an ORR of $18 \%$ by mRECIST and $14 \%$ by RECIST 1.1 in second-line treatment of advanced HCC (11). Thus, the conventional criteria (RECIST 1.1) may underestimate the effectiveness of immunotherapy. Our study found that the change in ORR between mRECIST (12 of 50, $24 \%$ ) and RECIST 1.1 (8 of 50,16\%) was $8 \%$ in patients with advanced HCC treated with PD-1 inhibitor. The relatively small sample size in the present research might have affected this result. A recent study has shown that a high ORR to nivolumab in phase II trials was associated with prolonged OS (11). Many studies have indicated that mRECIST criteria in HCC improved the sensitivity to quantify tumor response with targeted therapies (19) and patients who achieved a response on sorafenib had longer survival than nonresponders $(27,28)$. However, there is little research on the relationship between tumor response and survival in HCC patients treated with immunotherapy. Our study found that OS and PFS of the immunotherapy response group were significantly longer than those in the nonresponse group, which was classified by mRECIST $(p<0.05)$. Hence, our novel findings may suggest that mRECIST is better than RECIST 1.1 and iRECIST in determining immunotherapy response.

The mOS and mPFS of our study population were $20.9 \pm 12.5$ months and $11.5 \pm 10.2$ months based on follow-up data. The OS and PFS in our research were longer than previous studies for an immunotherapy monotherapy $(5,29)$. According to the Child-Pugh and ECOG scores of the patients included in our study, it showed that the patients were in good physical condition. The patients we included had fewer number that occurred macrovascular invasion (16\% vs.32\%) and less number of extrahepatic metastases $(64 \%$ vs.71\%) compared with CheckMate 040 study. In addition, most patients received further systemic treatments after anti-PD-1 discontinuation. These factors may lead to differences in patient survival analysis between our studies and other clinical studies.

This study attempted to demonstrate the intrareader variability of mRECIST in immunotherapy of HCC for the first time.

TABLE 5 | Compares overall survival (months) between patients with CR and PR and patients with SD and PD and between responders and nonresponders according to different response assessment methods.

\begin{tabular}{|c|c|c|c|c|c|c|}
\hline & CR & PR & $p$-value & SD & PD & $p$-value \\
\hline RECIST 1.1/iRECIST & $41.0 \pm 0$ & $24.6 \pm 9.8$ & 0.170 & $21.1 \pm 13.1$ & $20.2 \pm 12.9$ & 0.861 \\
\hline \multirow[t]{2}{*}{ mRECIST } & $32.0 \pm 10$ & $24.2 \pm 9.4$ & 0.204 & $19.3 \pm 8.2$ & $18.9 \pm 13.6$ & 0.915 \\
\hline & & Responders & & \multicolumn{3}{|c|}{ Nonresponders } \\
\hline RECIST 1.1/iRECIST & & $25.5 \pm 11.1$ & & \multicolumn{2}{|c|}{$20.7 \pm 13.0$} & 0.458 \\
\hline mRECIST & & $28.8 \pm 10.2$ & & \multicolumn{2}{|c|}{$19.0 \pm 12.5$} & 0.018 \\
\hline
\end{tabular}

$C R$, complete response; $P R$, partial response; $S D$, stable disease; $P D$, progressive disease. 
A

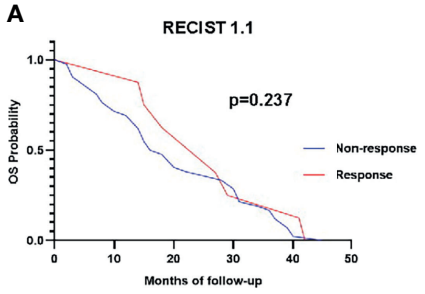

C

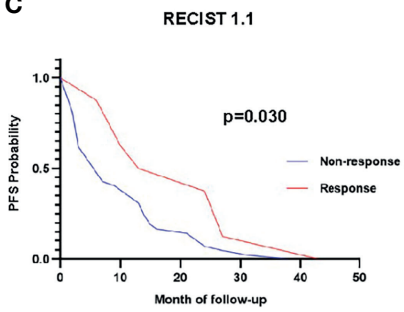

B

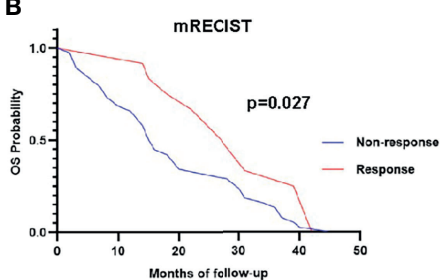

D

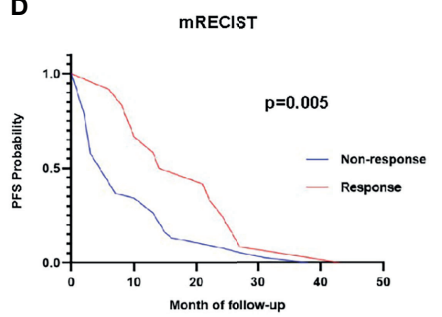

FIGURE 5 | Overall survival curve of responders (red line) versus nonresponders (blue line) using RECIST 1.1 (A) and mRECIST (B). Progression-free survival curve of responders (red line) versus nonresponders (blue line) using RECIST 1.1 (C) and mRECIST (D).

TABLE 6 | Compares PFS (months) between patients with CR and PR and patients with SD and PD and between responders and nonresponders according to different response assessment methods.

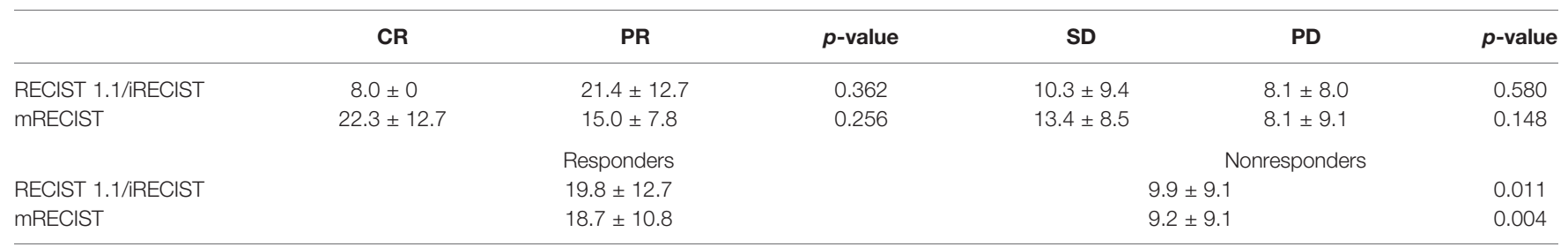

$C R$, complete response; $P R$, partial response; $S D$, stable disease; $P D$, progressive disease.

The concordance rate according to Cohen $\mathrm{k}$ was rather low compared with other papers (30). We suggest that the reason for the fair intrareader variability may be caused by the mRECIST measurement method which included measurement of the longest viable tumor diameter for the assessment of response. It is challenging for doctors to accurately measure the longest diameter of the viable tumor in lesions showing partial internal necrosis. Soft tissue resolution of CT is lower than MRI, leading to comparatively low disease detection and measurement sensitivity. Comparatively low disease detection and measurement sensitivity of CT could lead to deviation when determining tumor boundary and measuring tumor diameter. Furthermore, there may be a slight difference in the timing of each CT scan. The drugs used by patients

TABLE 7 | Compare the response assessment of two radiologists.

\begin{tabular}{lcc}
\hline & Kappa & Cl 95\% \\
\hline RECIST 1.1 & 0.534 & $0.305-0.763$ \\
iRECIST & 0.438 & $0.426-0.450$ \\
mRECIST & 0.363 & $0.351-0.375$
\end{tabular}

$\mathrm{Cl}$, confidence interval. in the two studies were different (PD-1 vs. sorafenib). Special therapeutic responses to tumor immunoassay inhibitor treatment, such as pseudo-progression and hyperprogress, etc., made it more difficult for radiologists with different qualifications. Thus, there is an urgent need for a more reasonable mRECIST assessment that provides a reliable method for assessing tumor response in HCC clinical trials. Quantitative imaging parameters related to immune efficacy evaluation are expected to be added to the mRECIST. Hence, our novel findings may indicate a disadvantage of mRECIST and provide new insights into mRECIST.

There were some limitations in this study. On the one hand, it was a retrospective study from a single center, resulting in recruitment bias. On the other hand, due to the limited number of patients treated with PD-1/PD-L1 inhibitor, further large-scale prospective studies are required to validate the results and examine the clinical utility of mRECIST in advanced HCC patients.

In conclusion, the immunotherapy-modified assessment method (mRECIST) has a powerful ability to discriminate between responders and nonresponders and shows significantly longer OS and PFS in responders than in nonresponders. However, it requires further improvements in order for it to be widely used in the clinical evaluation of immunotherapy in HCC. 


\section{DATA AVAILABILITY STATEMENT}

The original contributions presented in the study are included in the article/supplementary material. Further inquiries can be directed to the corresponding authors.

\section{ETHICS STATEMENT}

The studies involving human participants were reviewed and approved by Harbin Medical University Cancer Hospital. Written informed consent for participation was not required for this study in accordance with the national legislation and the institutional requirements. Written informed consent was not obtained from the individual(s) for the publication of any potentially identifiable images or data included in this article.

\section{AUTHOR CONTRIBUTIONS}

Conceived and designed the analysis: $\mathrm{TZ}$ and $\mathrm{YZ}$. Collected the data: MZ, YS, YX, JN, FW, and YH. Performed the analysis: MZ,

\section{REFERENCES}

1. Chiou V, Burotto M. Pseudoprogression and Immune-Related Response in Solid Tumors. J Clin Oncol (2015) 33(31):3541-3. doi: 10.1200/jco.2015.61.6870

2. Ayati N, Lee ST, Zakavi SR, Cheng M, Lau E, Parakh S, et al. Response Evaluation and Survival Prediction Following PD-1 Immunotherapy in Patients With Non-Small-Cell Lung Cancer: Comparison of Assessment Methods. J Nucl Med (2020) 62(7):926-33. doi: 10.2967/jnumed.120. 254508

3. Ribas A, Wolchok JJS. Cancer Immunotherapy Using Checkpoint Blockade. Science (2018) 359(6382):1350-5. doi: 10.1126/science.aar4060

4. Finn R, Ikeda M, Zhu A, Sung M, Baron A, Kudo M, et al. Phase Ib Study of Lenvatinib Plus Pembrolizumab in Patients With Unresectable Hepatocellular Carcinoma. J Clin Oncol (2020) 38(26):2960-70. doi: 10.1200/jco.20.00808

5. Rossi S, Toschi L, Castello A, Grizzi F, Mansi L, Lopci E, et al. Clinical Characteristics of Patient Selection and Imaging Predictors of Outcome in Solid Tumors Treated With Checkpoint-Inhibitors. Eur J Nucl Med Mol Imaging (2017) 44(13):2310-25. doi: 10.1007/s00259-017-3802-5

6. Mulkey F, Theoret MR, Keegan P, Pazdur R, Sridhara R. Comparison of iRECIST Versus RECIST V.1.1 in Patients Treated With an Anti-PD-1 or PDL1 Antibody: Pooled FDA Analysis. J ImmunoTher Cancer J Immunother Cancer (2020) 8:(1). doi: 10.1136/jitc-2019-000146

7. Eisenhauer E, Therasse P, Bogaerts J, Schwartz L, Sargent D, Ford R, et al. New Response Evaluation Criteria in Solid Tumours: Revised RECIST Guideline (Version 1.1). Eur J Cancer (2009) 45(2):228-47. doi: 10.1016/ j.ejca.2008.10.026

8. Llovet J, Lencioni R. mRECIST for HCC: Performance and Novel Refinements. J Hepatol (2020) 72(2):288-306. doi: 10.1016/j.jhep.2019.09.026

9. Lencioni R, Llovet J. Modified RECIST (mRECIST) Assessment for Hepatocellular Carcinoma. Semin Liver Dis (2010) 30(1):52-60. doi: 10.1055/s-0030-1247132

10. Seymour L, Bogaerts J, Perrone A, Ford R, Schwartz L, Mandrekar S, et al. iRECIST: Guidelines for Response Criteria for Use in Trials Testing Immunotherapeutics. Lancet Oncol (2017) 18(3):e143-52. doi: 10.1016/ s1470-2045(17)30074-8

11. El-Khoueiry A, Sangro B, Yau T, Crocenzi T, Kudo M, Hsu C, et al. Nivolumab in Patients With Advanced Hepatocellular Carcinoma (CheckMate 040): An Open-Label, Non-Comparative, Phase 1/2 Dose
YZ, CZ, SL, and YW. Drafting of the manuscript: TZ and MZ. Wrote the paper: MZ, TZ, CZ, and JN.

\section{FUNDING}

This study was supported by "Tou Yan Action" of Heilongjiang province, the National Natural Scientific Foundation of China (No. 81472322, No. 81872435, No. 81672930, and No. U20A20377); the Provincial Natural Science Foundation Outstanding Youth Project (JQ2019H003); the National Youth Talent Support Program for TZ; the Natural Science Foundation of Heilongiiang Province (LC201437/ H1617); the Fok Ying Tung Education Foundation (No. 151037); Scientific Research Project of the Health Planning Committee of Heilongjiang (2017130); the Academician Yu Weihan Outstanding Youth Foundation of Harbin Medical University for TZ; and the HaiYan Funds of Harbin Medical University for YZ (JZZD2020-17).

\section{ACKNOWLEDGMENTS}

We are thankful to the data providers for sharing their precious resources.

Escalation and Expansion Trial. Lancet (2017) 389(10088):2492-502. doi: 10.1016/s0140-6736(17)31046-2

12. Nishino M, Ramaiya N, Chambers E, Adeni A, Hatabu H, Jänne $P$, et al. Immune-Related Response Assessment During PD-1 Inhibitor Therapy in Advanced non-Small-Cell Lung Cancer Patients. J Immunother Cancer (2016) 4:84. doi: 10.1186/s40425-016-0193-2

13. Kim H, Heo M, Lee H, Sun J, Lee S, Ahn J, et al. Comparison of RECIST to Immune-Related Response Criteria in Patients With Non-Small Cell Lung Cancer Treated With Immune-Checkpoint Inhibitors. Cancer Chemother Pharmacol (2017) 80(3):591-8. doi: 10.1007/s00280-017-3396-4

14. Khoja L, Kibiro M, Metser U, Gedye C, Hogg D, Butler M, et al. Patterns of Response to Anti-PD-1 Treatment: An Exploratory Comparison of Four Radiological Response Criteria and Associations With Overall Survival in Metastatic Melanoma Patients. Br J Cancer (2016) 115(10):1186-92. doi: 10.1038/bjc.2016.308

15. Llovet J, Ricci S, Mazzaferro V, Hilgard P, Gane E, Blanc J, et al. Sorafenib in Advanced Hepatocellular Carcinoma. N Engl J Med (2008) 359(4):378-90. doi: 10.1056/NEJMoa0708857

16. Cheng A, Kang Y, Chen Z, Tsao C, Qin S, Kim J, et al. Efficacy and Safety of Sorafenib in Patients in the Asia-Pacific Region With Advanced Hepatocellular Carcinoma: A Phase III Randomised, Double-Blind, Placebo-Controlled Trial. Lancet Oncol (2009) 10(1):25-34. doi: 10.1016/ s1470-2045(08)70285-7

17. Cheng A, Kang Y, Lin D, Park J, Kudo M, Qin S, et al. Sunitinib Versus Sorafenib in Advanced Hepatocellular Cancer: Results of a Randomized Phase III Trial. Clin Oncol (2013) 31(32):4067-75. doi: 10.1200/jco.2012.45.8372

18. Johnson P, Qin S, Park J, Poon R, Raoul J, Philip P, et al. Brivanib Versus Sorafenib as First-Line Therapy in Patients With Unresectable, Advanced Hepatocellular Carcinoma: Results From the Randomized Phase III BRISK-FL Study. J Clin Oncol (2013) 31(28):3517-24. doi: 10.1200/jco.2012.48.4410

19. Llovet J, Montal R, Villanueva A. Randomized Trials and Endpoints in Advanced HCC: Role of PFS as a Surrogate of Survival. J Hepatol (2019) 70 (6):1262-77. doi: 10.1016/j.jhep.2019.01.028

20. Prieto J, Melero I, Sangro B. Immunological Landscape and Immunotherapy of Hepatocellular Carcinoma. Nat Rev Gastroenterol Hepatol (2015) 12 (12):681-700. doi: 10.1038/nrgastro.2015.173

21. Ringelhan M, Pfister D, O'Connor T, Pikarsky E, Heikenwalder M. The Immunology of Hepatocellular Carcinoma. Nat Immunol (2018) 19(3):22232. doi: $10.1038 / \mathrm{s} 41590-018-0044-\mathrm{z}$ 
22. Zhu A, Finn R, Edeline J, Cattan S, Ogasawara S, Palmer D, et al. Pembrolizumab in Patients With Advanced Hepatocellular Carcinoma Previously Treated With Sorafenib (KEYNOTE-224): A non-Randomised, Open-Label Phase 2 Trial. Lancet Oncol (2018) 19(7):940-52. doi: 10.1016/s1470-2045(18)30351-6

23. Therasse P, Arbuck S, Eisenhauer E, Wanders J, Kaplan R, Rubinstein L, et al. New Guidelines to Evaluate the Response to Treatment in Solid Tumors. European Organization for Research and Treatment of Cancer, National Cancer Institute of the United States, National Cancer Institute of Canada. J Natl Cancer Inst (2000) 92(3):205-16. doi: 10.1093/jnci/92.3.205

24. Queirolo P, Spagnolo F. Atypical Responses in Patients With Advanced Melanoma, Lung Cancer, Renal-Cell Carcinoma and Other Solid Tumors Treated With Anti-PD-1 Drugs: A Systematic Review. Cancer Treat Rev (2017) 59:71-8. doi: 10.1016/j.ctrv.2017.07.002

25. Miller A, Hoogstraten B, Staquet M, Winkler AJC. Reporting Results of Cancer Treatment. Cancer (1981) 47(1):207-14. doi: 10.1002/1097-0142 (19810101)47:1<207::aid-cncr2820470134>3.0.co;2-6

26. Fukuokaya W, Kimura T, Yanagisawa T, Kimura S, Tsuzuki S, Koike Y, et al. Comparison of the Immunotherapy Response Evaluation Criteria in Solid Tumours (iRECIST) With RECIST for Capturing Treatment Response of Patients With Metastatic Urothelial Carcinoma Treated With Pembrolizumab. BJU Int (2021) 127(1):90-5. doi: 10.1111/bju.15176

27. Edeline J, Boucher E, Rolland Y, Vauleon E, Pracht M, Perrin C, et al. Comparison of Tumor Response by Response Evaluation Criteria in Solid Tumors (RECIST) and Modified RECIST in Patients Treated With Sorafenib for Hepatocellular Carcinoma. Cancer (2012) 118(1):147-56. doi: 10.1002/cncr.26255

28. Ronot M, Bouattour M, Wassermann J, Bruno O, Dreyer C, Larroque B, et al. Alternative Response Criteria (Choi, European Association for the Study of the Liver, and Modified Response Evaluation Criteria in Solid Tumors [RECIST]) Versus RECIST 1.1 in Patients With Advanced Hepatocellular
Carcinoma Treated With Sorafenib. Oncologist (2014) 19(4):394-402. doi: 10.1634/theoncologist.2013-0114

29. Finn RS, Ryoo B-Y, Merle P, Kudo M, Bouattour M, Lim HY, et al. Pembrolizumab As Second-Line Therapy in Patients With Advanced Hepatocellular Carcinoma in KEYNOTE-240: A Randomized, DoubleBlind, Phase III Trial. J Clin Oncol (2020) 38(3):193-202. doi: 10.1200/ JCO.19.01307

30. Tovoli F, Renzulli M, Negrini G, Brocchi S, Ferrarini A, Andreone A, et al. Inter-Operator Variability and Source of Errors in Tumour Response Assessment for Hepatocellular Carcinoma Treated With Sorafenib. Eur Radiol (2018) 28(9):3611-20. doi: 10.1007/s00330-018-5393-3

Conflict of Interest: The authors declare that the research was conducted in the absence of any commercial or financial relationships that could be construed as a potential conflict of interest.

Publisher's Note: All claims expressed in this article are solely those of the authors and do not necessarily represent those of their affiliated organizations, or those of the publisher, the editors and the reviewers. Any product that may be evaluated in this article, or claim that may be made by its manufacturer, is not guaranteed or endorsed by the publisher.

Copyright (c) 2021 Zhou, Zhang, Nie, Sun, Xu, Wu, Huang, Li, Wang, Zhou and Zheng. This is an open-access article distributed under the terms of the Creative Commons Attribution License (CC BY). The use, distribution or reproduction in other forums is permitted, provided the original author(s) and the copyright owner(s) are credited and that the original publication in this journal is cited, in accordance with accepted academic practice. No use, distribution or reproduction is permitted which does not comply with these terms. 